\title{
Social organization in the Minority Game model
}

\author{
František Slanina \\ Institute of Physics, Academy of Sciences of the Czech Republic, \\ Na Slovance 2, CZ-18221 Praha, Czech Republic \\ e-mail: slanina@fzu.cz
}

\begin{abstract}
We study the role of imitation within the Minority Game model of market. The players can exchange information locally, which leads to formation of groups which act as if they were single players. Coherent spatial areas of rich and poor agents result. We found that the global effectivity is optimized at certain value of the imitation probability, which decreases with increasing memory length. The social tensions are suppressed for large imitation probability, but generally the requirements of high global effectivity and low social tensions are in conflict.
\end{abstract}

PACS numbers: 05.65.+b; 02.50.Le; 87.23.Ge

\section{INTRODUCTION}

The last decade witnessed the increasing effort of physicists to formulate various aspects of social sciences in the language of natural sciences, namely bring them close to physics. Among numerous applications we can mention for example traffic flow [1]2], city growth [3], human trail system [4], geometry of social networks [5] [6] economic returns of research [7] and so on.

Perhaps the most prominent example of this effort is the field of econophysics [8,9]. Among various attempts to grasp the nature of economic behavior the modeling of systems of heterogeneous adaptive agents reached high popularity. One of the basic models is the Minority Game [10,11] introduced by Challet and Zhang.

In the Minority Game (MG) we have $N$ players who choose repeatedly between two options and compete to be in the minority group. This is the idealization of various situations, where the competition for limited resources leads to intrinsic frustration. One can think for example of cars choosing between two alternative routes or a speculator who tries to earn money by buying and selling shares in such a manner that the majority takes the opposite action than herself.

The players share a public information, saying what were the outcomes of the game in past $M$ rounds. The players interact only through this information. Therefore, the system has a "mean-field" character, in the sense that no short-range interactions exist.

The self-organization is achieved by allowing players to have several strategies and choose among them the strategy which seems to be the best one. This feature leads to decrease of the fluctuations of attendance below its random coin-tossing value, thus increasing the global effectivity of the system. It was found that the relevant parameter is $\alpha=2^{M} / N$ and the maximum effectivity is reached for $\alpha=\alpha_{c} \simeq 0.34$ [11 13].

It was established that a phase transition occurs at this value of the parameter $\alpha$ and its properties were thoroughly studied both numerically [14] and analytically
[15. The Minority Game and its variants were intensively studied by several groups. We are able to give only an incomplete list of references 16 26]. Among the various ramifications, we would like to mention especially the attempts to go back to the economic motivations of MG and model the market mechanisms 2729$]$.

The purpose of the present work is to investigate the properties of social structures, which can emerge if we go beyond the mean-field character of the usual MG and allow a local information exchange. Related works were already done, either assuming that the global information is fully replaced by a local one 30] or using the MG scheme for evolving the Kauffmans's boolean networks 31] to the critical state [32.

Here we want to study the effect of imitations. Indeed, it is quite common that people do not invest individually, but rely on an advice from specialized agencies, or simply follow the trend they perceive in their information neighborhood. In so doing, the individuals coalesce into groups, which act as single players. In the framework of Minority Game, we will study the social structure induced by the occurence of these groups.

\section{MINORITY GAME WITH IMITATION}

We introduce the possibility of local information exchange in our variant of the Minority Game. To this end, we should assume some properties of the information network underlaying the "information metabolism", in analogy to the metabolic pathways in living organisms. The study of the geometry of information networks is now a scientific field on its own [5,6, 33]. Within the framework of MG a linear chain [30] and random network with fixed connectivity $K$ [32] was already investigeted in different contexts. Here we assume the simplest geometry, namely we will place the players on a linear chain with nearest-neighbor connections. We will simplify the network even more by suposing, that the information flow is not symmetric and each player can obtain information only from her left-hand neighbor. In the rest of 
the article, by "neighbor" we always mean the left-hand neighbor. Each player can read the information about her neighbors' wealth.

There wil be two conditions needed for a player to imitate her neighbor. First, the player should have internal disposition for being an imitator. We simplify the variety of risk-aversion levels by postulating only two types of players. The imitator type (denoted 1) will always adopt the strategy of her neighbor, if she finds it better. The rest consists of the leader type (denoted 0) who always uses her own strategies. In the whole population of $N$ players, there is the fraction $p \in[0,1]$ of the type 1 and the rest is of the type 0 . The case $p=0$ corresponds to the usual MG.

The second condition for the player of the type 1 to actually imitate in the current step is that her neighbor has larger accumulated wealth than the player itself. We suppose that the played does not know what are the strategie of her neighbor, but if she observes that the neighbor's behavior is more profitable than her own strategy, she relegates the decision to the neighbor and takes the same action. The player of the type 0 will never imitate. Therefore, she will always look only at her $S$ strategies and choose the best estimate from them.

The above rules are formalized as follows. We follow mostly the notation of 15$]$. There are totally $N$ players. We will take always $N$ as an odd number. Each player has $S=2$ strategies, denoted $s_{j} \in\{1,2\}$.

We denote the two possible actions a player can take +1 and -1 . The winning action is +1 if most players took -1 and vice versa. The players know the last $M$ outcomes of the game. This information is arranged into the $M$-bit string $\mu \in\{-1,+1\}^{M}$. The strategies are tables attributing to each of $2^{M}$ possible strings $\mu$ the action $a_{j, s_{j}}^{\mu}$ the player $j$ takes, if she chooses the strategy $s_{j}$. The scores $U_{j, s}$ of the strategies are updated according to the minority rule

$$
U_{j, s}(t+1)=U_{j, s}(t)-a_{s, j}^{\mu(t)} \operatorname{sign} \sum_{i} a_{i}(t)
$$

where $a_{j}(t)$ is the action the player $j$ takes at time $t$.

Up to this moment the algoritm was identical to the usual MG. We introduce a new feature, the imitation, into the rule determining what are the actions taken by the players, depending on their wealths and on the scores of their strategies.

Each player has a label $\tilde{l} \in\{1,0\}$ indicating, whether the player is potentially an imitator $(\tilde{l}=1)$ or always a leader $(\tilde{l}=0)$. The labels are random but quenched. At the beginning we take each of the players and attribute her the label 1 with probability $p$ and label 0 with probability $1-p$.

Denote $W_{j}$ the wealth of the $j$-th player. Then, if the label $\tilde{l}_{j}=1$ and $W_{j-1}>W_{j}$, then $j$ will take the same action as $j-1$, so $a_{j}=a_{j-1}$. Therefore, wee need to establish $a_{j-1}$ first and we proceed recurrently by iterating this rule. On the other hand, if $\tilde{l}_{j}=0$ or $W_{j-1} \leq W_{j}$, the action will be $a_{j}=a_{j, s_{\mathrm{M}}}$, where $s_{\mathrm{M}}$ denotes the most successfull strategy at the moment, $U_{j, s_{\mathrm{M}}}=\max _{s} U_{j, s}$, as in the usual MG.

This can be written formally by introducing the variables $l_{j}$ describing the actual state of imitation, in analogy with the labels $\tilde{l}_{j}$ describing potential state of imitation. We can write $l_{j}=\tilde{l}_{j} \theta\left(W_{j-1}-W_{j}\right)$, with $\theta(x)=1$ for $x>0$ and 0 othervise. The actions are

$$
a_{j}=l_{j} a_{j-1}+\left(1-l_{j}\right) a_{j, s_{\mathrm{M}}} .
$$

We also suppose that the imitation is not for free. The player who imitates passes a small fraction $\varepsilon$ of its wealth increase to the imitated player. This rule accounts for the price of information. We used the value $\varepsilon=0.05$. Then, we update the wealth of players iteratively,

$$
\Delta W_{j}(t)=\left(1-\varepsilon l_{j}\right)\left(\varepsilon l_{j+1} \Delta W_{j+1}(t)-a_{j} \operatorname{sign} \sum_{i} a_{i}(t)\right)
$$

where $\Delta W_{j}(t)=W_{j}(t+1)-W_{j}(t)$.

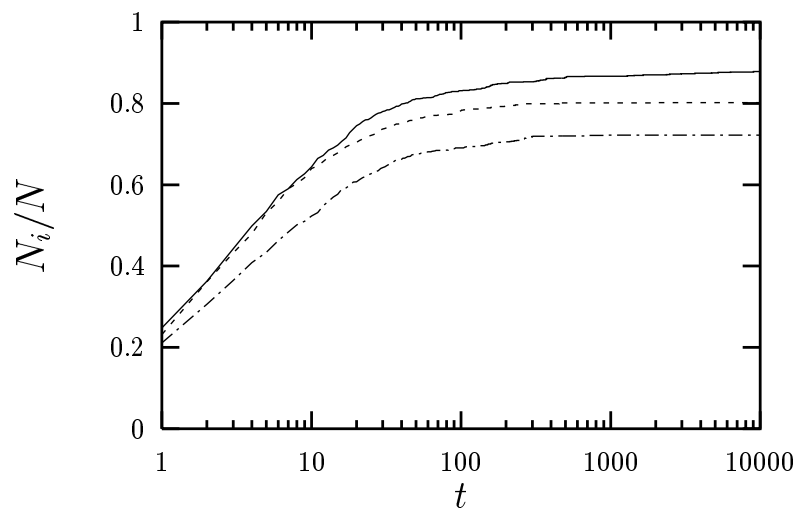

FIG. 1. Time dependence of the fraction of imitating players for the number of players is $N=1001$ and memory $M=6$. The imitation probabilities $p$ are 0.99 (solid line), 0.95 (dashed line), and 0.8 (dash-dotted line).

In numerical simulations we observe that the number of actually imitating players, $N_{i}=\sum_{j} l_{j}$, grows until it saturates at a value slightly below the upper bound $p N$. It means that it is always beneficiary for some potential imitators not to imitate but rely on their own strategies. The time dependence of the fraction of imitators $N_{i} / N$ for three values of $p$ is shown in Fig. 11.

The initially random distribution of wealth among players changes qualitatively during the evolution of the system. Coherent groups of poor and wealthy players are formed. An example of the time evolution of the spatial wealth distribution is given in Fig. 22. 


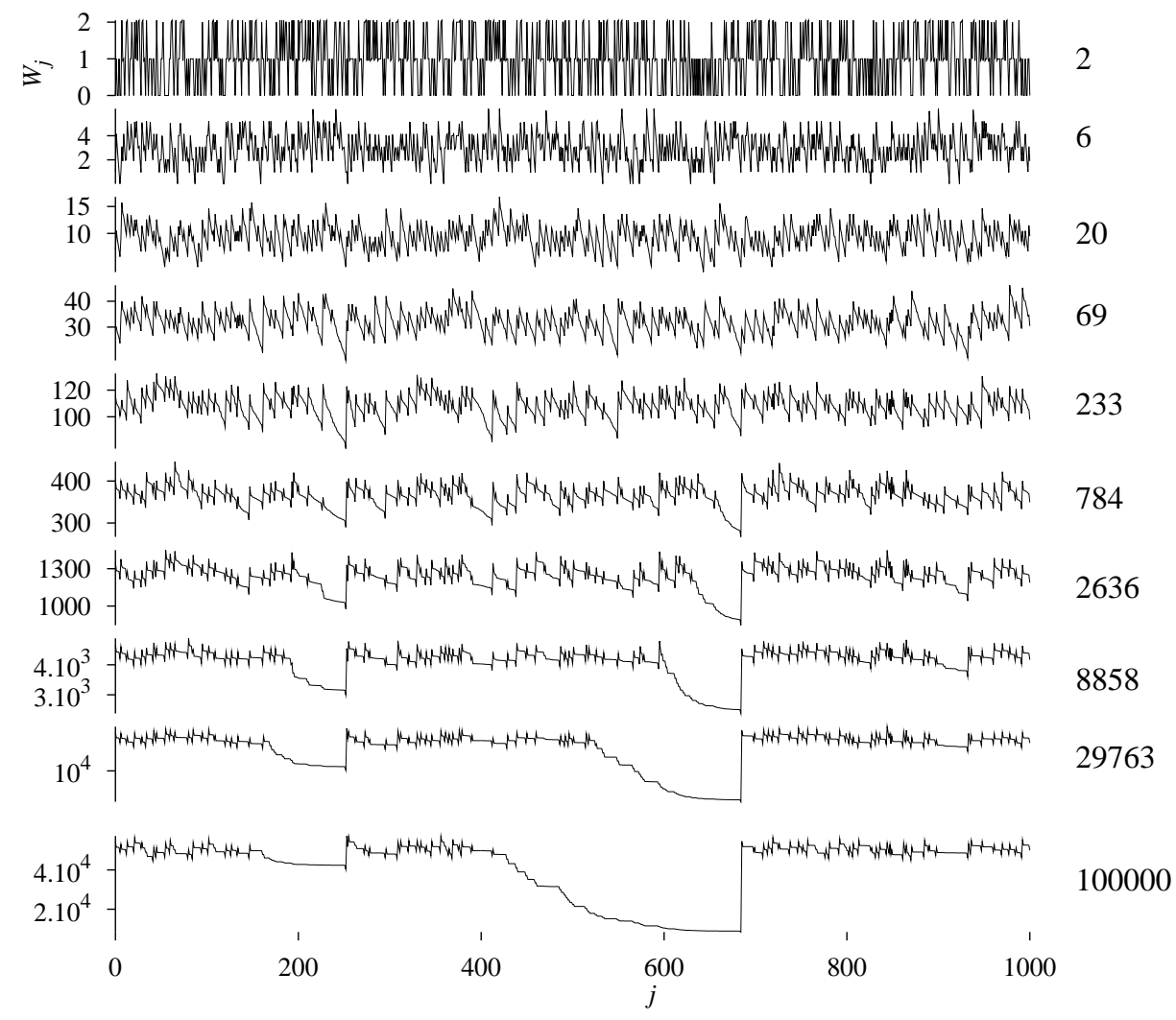

FIG. 2. Example of the evolution of the distribution of wealth among players, for $p=1$. The time step at which the snapshot is taken is indicated on the right. For each time, the vertical axis indicates the wealth $W_{j}$ of the $j$ 'th player.

\section{GLOBAL EFFECTIVITY AND LOCAL SOCIAL TENSIONS}

The Minority Game is a negative-sum game. The average gain of the players reaches its maximum, if the attendance $A=\sum_{j} \frac{1}{2}\left(a_{j}+1\right)$ is closest possible to $N / 2$. So, the time averaged attendance fluctuations $\sigma^{2}=\left\langle(A-N / 2)^{2}\right\rangle$ measure the distance from the global optimum. The global effectivity is higher for smaller $\sigma^{2}$.

We investigated the influence of the imitation on the global effectivity. In the usual MG (which corresponds to $p=0$ ) the fluctuations depend on the memory and number of players through the scaling variable $\alpha=2^{M} / N$. This fact can be observed through the data collapse of the results for various $N$ 's. We tried to find the same data collapse for a non-zero $p$. Figure 3 shows such a plot for $p=0.8$. We can see that the dependence of $\sigma^{2} / N$ on $\alpha$ has a minimum as in the usual MG, but the critical value $\alpha_{c}$ of the scaling parameter is lower while the value of the flucuations at the minimum is higher. In the symetry-broken phase $\left(\alpha>\alpha_{c}\right)$ the data collapse is good. On the other hand, significant deviations are observed in the crowded (symmetric) phase, which are more pronounced for smaller $\alpha$. On the other hand, for larger value of imitation probability, the data still scale with $\alpha=2^{M} / N$, but the collapse is poorer in the whole range of $\alpha$. The situation for $p=0.99$ is shown in Fig. 4. We can see that if we decrease $\alpha$ in the symmetric phase, the fluctuations increase first, but contrary to the usual MG a local maximum is reached and when decreasing $\alpha$ further, the fluctuations are suppressed.

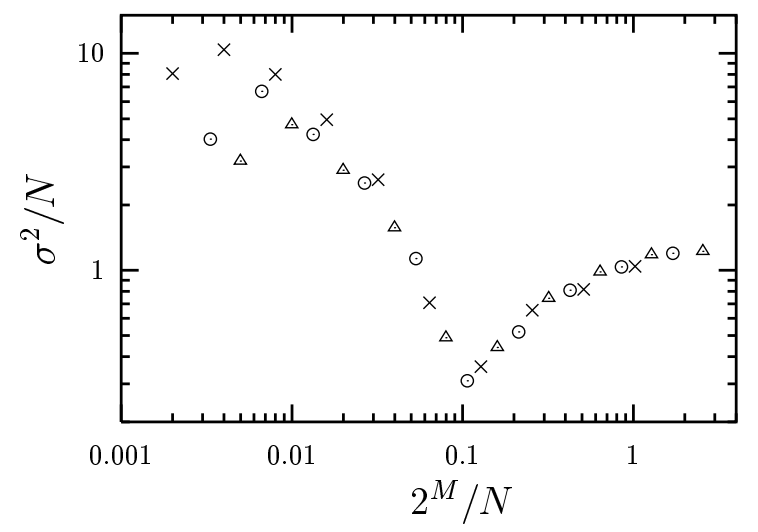

FIG. 3. Dependence of the attendance fluctuations on the scaling variable $2^{M} / N$, for $p=0.8$ and system sizes $N=1001$ $(\times), 601(\odot)$, and $401(\triangle)$. 


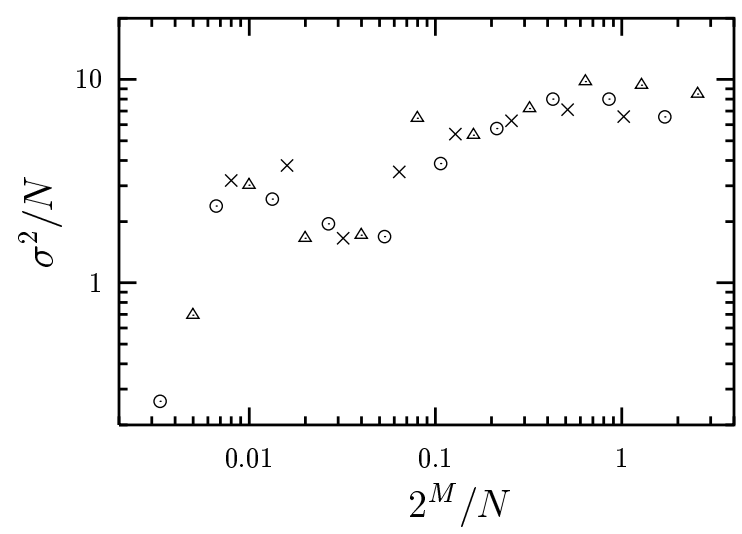

FIG. 4. Dependence of the attendance fluctuations on the scaling variable $2^{M} / N$, for $p=0.99$ and system sizes $N=1001(\times), 601(\odot)$, and $401(\triangle)$.

We tried also another data collapse, namely the dependence of $\sigma^{2} / N s_{\sigma}$ on $2^{M} s_{M} / N$ for various $p$, where $s_{M}$ and $s_{\sigma}$ are scaling parameters depending on $p$, chosen so that the best possible data collapse is achieved. For convenience we require $s_{M}=s_{\sigma}=1$ for $p=0$. With this choice, the scaling parameters have the following interpretation. The location minimum of attendance fluctuations is shifted from its usual value $\alpha_{c}(0)=0.34$ valid for $p=0$ to $\alpha_{c}(p)=\alpha_{c}(0) / s_{M}$, while the value of $\sigma^{2} / N$ in the minimum is increased by the factor $s_{\sigma}$. We can see the results in Fig. 5. We again observe good collapse in the symmery-broken regime, while in the symmetric phase there is no collapse, but systematic decrease, stronger for higher $p$, is observed.

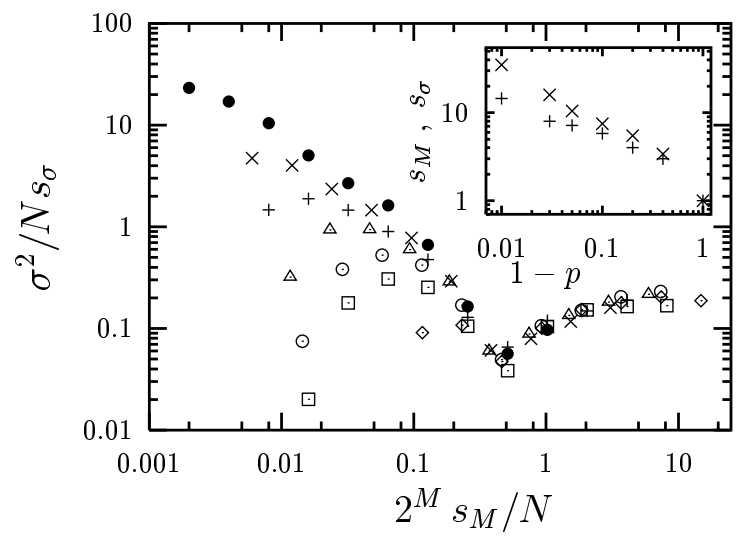

FIG. 5. Rescaled atendance fluctuations. The number of players is $N=1001$ and the imitation probabilities $p$ are 0 $(\bullet), 0.6(\times), 0.8(+), 0.9(\triangle), 0.95(\odot), 0.97(\square)$, and 0.99 $(\diamond)$. Inset: dependence of the scaling parameters $s_{M}(+)$ and $s_{\sigma}(\times)$ on $p$.

Nevertheless, the parameters $s_{M}$ and $s_{\sigma}$ still bear the information about the shift of the minimum. We can see their dependence on $p$ in the inset of Fig. 5. The data suggest that both of the parameters diverge as a power law when $p \rightarrow 1$.
We investigated also the dependence of the attendance fluctuations (and therefore the global effectivity) on the imitation probability for fixed number of players and memory. We found that in the crowded phase the system becomes more effective if imitation is allowed $(p>0)$, but there is a local minimum in the dependence of $\sigma^{2} / N$ on $p$, indicating that there is an optimal level of imitation, beyond which the system starts to perform worse. The results for $N=1001$ are shown in Fig. 6. We can see that the minimum occurs at smaller values for larger $M$. We can also observe that for longer memories $(M=7$ in our case) the value of the fluctuations for $p=1$ is significantly above the value vithout imitation $(p=0)$, while the value at the minimum still lies below the $p=0$ value. This implies that moderate imitation can be beneficiary, while exaggerated one can be harmful.

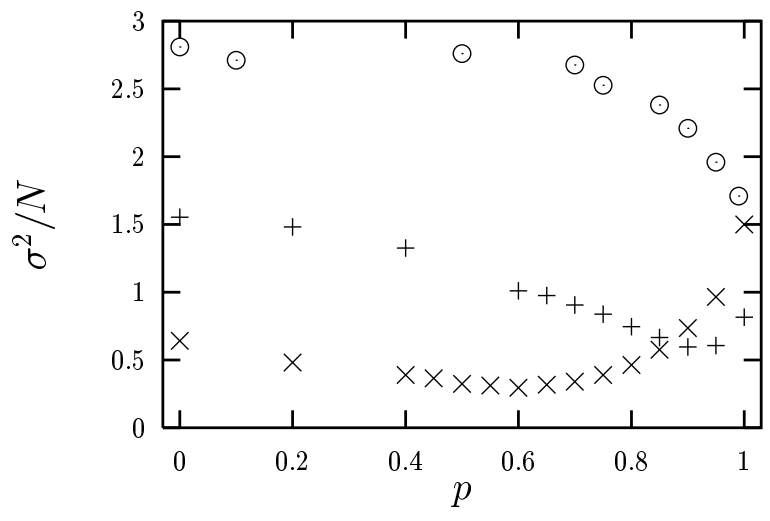

FIG. 6. Dependence of the attendance fluctuations on the imitation probability. The number of players is $N=1001$ and memory length $M=5(\odot), M=6(+)$, and $M=7(\times)$.

The increase of spatial coherence in poor and wealthy groups seen qualitatively in Fig. 2 can result in decrease of social tension. Indeed, the individuals can exchange information about their wealth only among neighbors. If most of the individuals perceive small local wealth gradient, the social tensions will be also small. To quantify the social tension, we should introduce a sort of "utility function" 34] $U(\Delta W)$, which indicates, how much the wealth difference $\Delta W$ is subjectively perceived. As usual, any realistic utility function should have negative second derivative. This feature, called risk aversion in standard economics literature, expresses in our case the intuitively obvious fact that smaller differences in living standards are relatively more significant than larger differences: I could be more happy if I succeeded in shrinking the wage distance to my rival employee from $500 \$$ to $400 \$$ than from $55,500 \$$ to $55,400 \$$. 
We will use the utility function in the form $U(x)=x^{b}$. Realistic values of the exponent are $b<1$. However, we will use also the values $b=1$ and $b=2$ for comparison. Then, the global measure of the local social tension is

$$
d_{b}=\frac{1}{\langle W\rangle}\left(\sum_{j=1}^{N-1}\left|W_{j}-W_{j+1}\right|^{b}\right)^{1 / b}
$$

where we denoted the average wealth $\langle W\rangle=\frac{1}{N} \sum_{j=1}^{N} W_{j}$.

The results for various values of the exponent $b$ are shown in Fig. 7. We can see that the behavior for all $b$ except $b=2$ is qualitatively similar. When increasing $p$, the social tension increases first, but then decreases again and for $p \rightarrow 1$ it reaches a point below its value for $p=0$. Hence, large enough imitation will decrease the social tension. On the other hand, for $b=2$ we observe further increase when $p$ approaches to 1 . Therefore, if the utility was measured by exponent $b=2$ (which would hold for risk-seeking adventurers), the tension would only increase due to imitation.

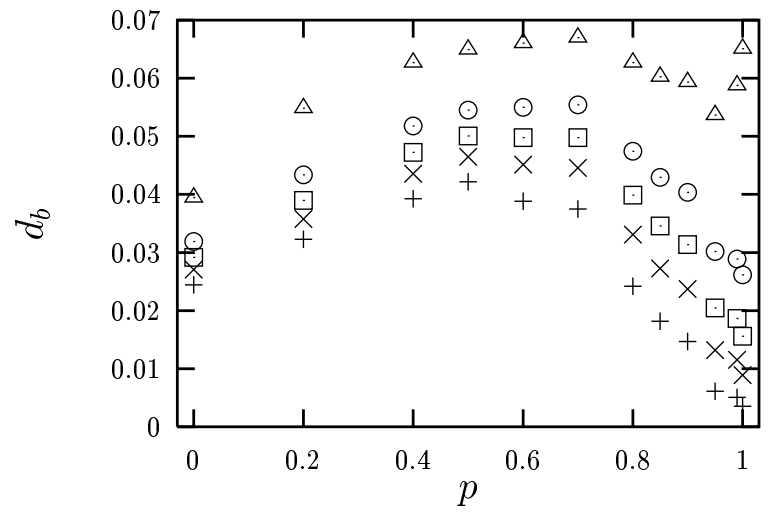

FIG. 7. Relative local tension for $N=1001, M=6$, measured by utility function $(\Delta W)^{b}$ with exponents $b=0.3(+)$, $b=0.5(\times), b=0.7(\square), b=1(\odot)$, and $b=2(\triangle)$.

The most striking result comes from comparison of Fig 6 (for $M=6$ ) and Fig 7 . We can see that both the local maxima of $\sigma^{2} / N$ and the local minima of $d_{b}$ for $b<1$ lie at the endpoints of the interval $[0,1]$. Therefore, if we want to optimize the social tensions by some process invoving small gradual changes of $p$, we will finish in one of the endpoints, depending on the initial value of $p$. Among the two stable points, the optimum is located at $p=1$.

However, the stable points (including the absolute optimum of the local tension) are simultaneously local minima of the global effectivity: we observe a clear conflict of interest between local and global views.

\section{CONCLUSIONS}

We investigated the creation of rich and poor spatial domains due to local information exchange, within the framework of the Minority Game. The introduction of imitation among players leads to creation of groups, which act in accord. For fixed imitation probability $p$ we found that the attendance fluctuations still depend on the scaling variable $\alpha=2^{M} / N$ in the symmery-broken phase, while in the symmetric phase there are systematic deviations from the data collapse.

The characteristic minimum in the dependence of fluctuations on $\alpha$ persist in our modification of the Minority Game, but the location of the minimum decreases and the value at the minimum increases when increasing $p$. We found that both quantities depend as a power law on $1-p$.

We found that in the symmetric phase the introduction of imitation can lead to absolute decrease of the attendance fluctuations and thus to increase in global system effectivity. There is an optimal value of the imitation, which increases with decreasing $M$.

The creation of coherent areas of poor and rich agents leads to decrease in the local social tensions, but only if $p$ is sufficiently close to 1 . The lowest value of the social tension is reached at $p=1$, but for such a value the global effectivity is significantly lower than its optimum value. Therefore, we observe a conflict of local interests (minimization of social tension) with global performance (minimization of attendance fluctuations).

\section{ACKNOWLEDGMENTS}

I am indebted to Yi-Cheng Zhang for numerous useful discussions. I acknowledge the financial support from the University of Fribourg, Switzerland, where part of this work was done.

[1] K. Nagel and M. Schreckenberg, J. Phys. I France 2, 2221 (1992).

[2] K. Nagel and M. Paczuski, Phys. Rev. E 51, 2909 (1995).

[3] M. Marsili and Y.-C. Zhang, Phys. Rev. Lett. 80, 2741 (1998).

[4] D. Helbing, J. Keltsch, and P. Molnár, Nature 388, 47 (1997).

[5] D. J. Watts and S. H. Strogatz, Nature 393, 440 (1998).

[6] A.-L. Barabási and R. Albert, Science 286, 509 (1999).

[7] D. Sornette and D. Zajdenweber, Eur. Phys. J. B 8, 653 (1999).

[8] R. N. Mantegna and H. E. Stanley, Introduction to Econophysics: Correlations and Complexity in Finance (Cambridge University Press, Cambridge, 1999).

[9] J.-P. Bouchaud and M. Potters, Theory of Financial Risks (Cambridge University Press, Cambridge, 2000).

[10] D. Challet and Y.-C. Zhang, Physica A 246, 407 (1997).

[11] D. Challet and Y.-C. Zhang, Physica A 256, 514 (1998). 
[12] R. Savit, R. Manuca, and R. Riolo, Phys. Rev. Lett. 82, 2203 (1999).

[13] N. F. Johnson, S. Jarvis, R. Jonson, P. Cheung, Y. R. Kwong, and P. M. Hui, Physica A 258, 230 (1998).

[14] D. Challet and M. Marsili, Phys. Rev. E 60, R6271 (1999).

[15] D. Challet, M. Marsili, and R. Zecchina, Phys. Rev. Lett. 84, 1824 (2000).

[16] M. A. R. de Cara, O. Pla, and F. Guinea, Eur. Phys. J. B 10, 187 (1999).

[17] A. Cavagna, J. P. Garrahan, I. Giardina, and D. Sherrington, Phys. Rev. Lett. 83, 4429 (1999).

[18] A. Cavagna, Phys. Rev. E 59, R3783 (1999).

[19] N. F. Johnson, P. M. Hui, D. Zheng, and M. Hart, J. Phys. A: Math. Gen. 32, L427 (1999).

[20] D. Challet and M. Marsili, cond-mat/0004196.

[21] N. F. Johnson, P. M. Hui, R. Jonson, and T. S. Lo, Phys. Rev. Lett. 82, 3360 (1999).

[22] N. F. Johnson, P. M. Hui, D. Zheng, and C. W. Tai, Physica A 269, 493 (1999).

[23] H. Ceva, Physica A 277, 496 (2000).

[24] R. D'hulst and G. J. Rodgers, Physica A 278, 579 (2000).

[25] N. F. Johnson, M. Hart, P. M. Hui, cond-mat/9811227.

[26] M. Hart, P. Jefferies, N. F. Johnson, and P. M. Hui, cond-mat/0003486.

[27] D. Challet, M. Marsili, and Y.-C. Zhang, Physica A 276, 284 (2000).

[28] F. Slanina and Y.-C. Zhang, Physica A 272, 257 (1999).

[29] N. F. Johnson, M. Hart, P. M. Hui, and D. Zheng, condmat/9910072

[30] T. Kalinowski, H.-J. Schulz, and M. Briese, Physica A 277, 502 (2000).

[31] S. A. Kauffman, The Origins of Order: Self-organization and Selection in Evolution (Oxford University Press, Oxford, 1993).

[32] M. Paczuski and K. E. Bassler, Phys. Rev. Lett. 84, 3185 (2000).

[33] F. Slanina and M. Kotrla, cond-mat/0004407.

[34] R. C. Merton, Continuous-time finance (Blackwell publishers, Cambridge, 1990). 